\title{
EDITORIAL - MIGRATIONS- UND FLUCHTERFAHRUNGEN ERZÄHLEN UND ERINNERN
}

\author{
Anna Schnitzer \& Rebecca Mörgen
}

Grenzüberschreitende Migrationsbewegungen stellen keineswegs neue Entwicklungen dar, sondern sind in historischer Perspektive auch für europäische Gesellschaften schon lange konstitutiv. Dabei sind Migrationsgesellschaften durch komplexe Differenz- und Ungleichheitsverhältnisse geprägt, ein Neben- und Miteinander verschiedener Sprachen und Mehrfachzugehörigkeiten sowie unterschiedliche Zuschreibungen und Diskriminierungs- sowie Ausgrenzungserfahrungen.

Migrationsprozesse gehen mit Erfahrungen von Brüchen, Neuorientierungen und der Suche nach Kontinuitäten einher. Die Lebenssituation ist insbesondere für Menschen mit Fluchtgeschichte noch lange nach ihrer Ankunft im europäischen Migrationsregime von strukturell bedingten Unsicherheiten geprägt, wie bspw. unsicherem Aufenthaltsstatus, Nicht-Anerkennung von Bildungsabschlüssen oder auch fehlender Lohnarbeit. Gleichzeitig werden diese strukturell bedingten Unsicherheiten, die Verletzbarkeiten hervorbringen können, von den Akteur*innen auf eigensinnige Art und Weise bearbeitet und es werden Handlungsräume eröffnet.

Entsprechend können Migrationsbewegungen und Migrationsprozesse für die Akteur*innen sowohl mit Verlusterfahrungen, Gefühlen des Scheiterns als auch der Eröffnung von Möglichkeitsräumen - und damit mit einem produktiven Verknüpfungspotential von „Neuem“ und „Fremden" - einhergehen. Migrationsprozesse bringen zudem Veränderungen des sozialen Beziehungsgeflechts mit sich, bspw. im Aufbau transnationaler Netzwerke, Irritationen gewohnter Praktiken sowie Relevanzsetzungen biographischen Wissens. Doch wie wird Migrationsgeschichte erinnert und was bleibt dabei im Verborgenen? Wie lässt sich über kollektive Erfahrungen von Migrationsbewegungen und 
teur*innen in den Blick. Dabei stehen die verschiedenen Praktiken im Fokus, mit denen Migrationsgeschichte(n) in ihren Verläufen erinnert und in ihren Transformationsprozessen erzählt werden. Das Heft versammelt Beiträge, die aktuelle empirische Ergebnisse vorstellen und methodische sowie methodologische Herausforderungen thematisieren, die im Zuge der Forschung bearbeitet werden.

Im ersten Beitrag des Thementeils setzt sich Lena Inowlocki mit der Frage auseinander, wie Migration, Verfolgung und Flucht zwischen Generationen in Familien erzählt und erinnert wird. Sie nutzt in ihrer Analyse das Konzept des szenischen Verstehens, um biographische Leidenserfahrungen als Herausforderungen der Thematisierung von Erinnerungen zwischen den Generationen herauszuarbeiten. Dabei zeigt sie, in welcher Weise zeitgeschichtliche, institutionelle, politische und kollektive Dimensionen der Erfahrungen Erinnerungsprozesse und die Möglichkeiten des Erzählens über Erlebtes in den Familien beeinflussen. Empirische Basis ihrer Argumentation sind zum einen Forschungssituationen eigener Forschungsprojekte, zum anderen literarische Beispiele der (De-)Thematisierung von Erinnerungen in der Familie.

Lalitha Chamakalayil, Oxana Ivanova-Chessex, Bruno Leutwyler und Wiebke Scharathow stellen in ihrem Beitrag das biographische Erzählen einer Migrationsgeschichte in einen machtvollen gesellschaftlichen Kontext. Dabei gehen sie nicht nur von einer Determinierung der Erzählungen durch gesellschaftliche Bedingungen aus, die gleichwohl wirksam werden. Vielmehr nehmen sie mit einer biographie- und subjektivierungstheoretischen Perspektive die eigensinnige Gestaltung der Erzählung der eigenen Fluchterfahrung genauer in den Blick. Wie lässt sich über die eigene Fluchterfahrung im Kontext migrationsgesellschaftlicher und neoliberaler Ordnungen erzählen? Anhand einer Fallanalyse gehen die Autor*innen dieser Frage nach, und zeigen auf, welche „Räume der Eigensinnigkeit des biographischen Erzählens“ sich (nicht) eröffnen.

In ihrem englischsprachigen Beitrag untersucht Dilyara Müller-Suleymanova, in welcher Weise
Jugendliche der zweiten Generation bosnischer Herkunft im Interview einen Bezug zur konfliktgeprägten Lebensgeschichte ihrer Eltern herstellen. Wie wird der Konflikt im Herkunftsland der Eltern in den Interviews thematisiert und zum Gegenstand der eigenen Lebensgeschichte und Lebensplanung gemacht? Dieser Frage geht die Autorin auf der Basis narrativ-biographischer Interviews nach. Dabei zeigt sie insbesondere die aktive Rolle auf, die die Angehörigen der zweiten Generation in der Auseinandersetzung mit der politischen Vergangenheit und der Konfliktgeschichte des Herkunftslandes der Eltern einnehmen. Anhand einer exemplarischen biographischen Fallanalyse einer jungen Frau wird deutlich, wie diese in Auseinandersetzung mit den bruchstückhaften Erzählungen der Eltern, in Abgrenzung zu deren Positionierungen und im Austausch mit jungen Menschen ihrer Generation ihre eigenen Sichtweisen entwickelt.

Im Beitrag von Miriam Friz Trzeciak wechselt die Perspektive von lebensgeschichtlichen hin zu kollektiven Erinnerungen von Migrationsbewegungen. Basierend auf einem Lehrforschungsprojekt wird die Vernachlässigung bzw. die Dethematisierung des kolonialen Erbes deutscher Städte problematisiert. Gegenstand des Artikels sind „multidirektionale Formen der Erinnerung", die anhand einer postkolonialen und postsozialistischen Stadtführung in Cottbus rekonstruiert werden. Der Beitrag kontextualisiert die eigenen Erkenntnisse mit Befunden der memory studies sowie der post- und dekolonialen Theoriebildung und stellt die These auf, dass mit multidirektionalen Formen der Erinnerung rechtsextremen und rassistischen Narrativen begegnet werden kann, indem gemeinsam Gegennarrative hervorgebracht werden. Hierbei zeigt der Beitrag auf, dass Orte mit umkämpften Erinnerungen einhergehen, die jedoch nicht zwangsläufig Konkurrenz um Anerkennung zwischen sozialen Gruppen reproduzieren müssen. In der Sichtbarmachung der verschiedenen Geschichten liegt für den*die Autor*in das Potential für neue Formen von Solidaritäten.

Im letzten Beitrag des Heftes nimmt Anna Schnitzer wie auch bereits Lena Inowlocki Erinnerungspraktiken in Familien mit Migrationsgeschichte in den Blick und geht der Frage nach, 
wie sich die eigene Familiengeschichte erzählen lässt. Während Lena Inowlocki sich Familienerinnerungen über das Konzept des szenischen Verstehens und sensibilisierende Bezüge zu künstlerisch-literarischen Darstellungen nähert, argumentiert Anna Schnitzer für eine methodologisch begründete Verschränkung ethnographischer und biographischer Zugänge. So wird es möglich, in verschiedenen Erhebungssituationen erzählte kleine Geschichten und situative Beobachtungen zu einem nachvollziehenden Verstehen familiärer Erinnerungspraktiken zu verschränken.

In einem freien Beitrag berichten zudem Susanne Benzel und Vera King von dem Promotionskolleg „Psychosoziale Folgen von Migration und Flucht - generationale Dynamiken und adoleszente Verläufe", das noch bis November 2021 an den Universitäten Frankfurt, Hamburg, Kassel und Tübingen durchgeführt und von der Hans-Böckler-Stiftung gefördert wird. Insgesamt neun geförderte Dissertationsprojekte widmen sich der interdisziplinären Erforschung der „psychosozialen und psychischen Folgen von Migration und Flucht und deren Verarbeitung und Bewältigung angesichts prekärer gesellschaftlicher Rahmenbedingungen“. Dabei stehen insbesondere Eltern-Kind-Beziehungen und adoleszente
Generationendynamiken sowie transgenerationale Transmissionsprozesse im Zentrum der Forschung.

Der Themenschwerpunkt dieses Heftes geht auf eine Tagung zurück, die im Herbst 2019 an der Universität Zürich durchgeführt wurde. Wir möchten an dieser Stelle ganz herzlich allen danken, die die Tagung im Herbst 2019 möglich gemacht haben. Neben dem ganzen Team des Lehrstuhls Ausserschulische Bildung und Erziehung des Instituts für Erziehungswissenschaft der Universität Zürich und allen Referent*innen gebührt unser Dank vor allem Ellen Höhne, die die Tagung maßgeblich mitkonzipiert und -organisiert hat. Zudem danken wir dem ganzen GISoTeam für die kontinuierliche Unterstützung und Beratung im Zuge der Realisierung dieses Heftes. Daniel Werner hat alle Beiträge dieses Heftes aufmerksam gelesen und mit viel sprachlichem Feingefühl lektoriert. Dafür danken wir ihm herzlich.

Wir hoffen, Ihnen mit diesem Heft Einblicke in aktuelle Forschungsarbeiten zu Fragen der Erinnerung und Erzählung von Migrations- und Fluchterfahrungen zu geben und wünschen eine anregende Lektüre!

\section{Die Redaktion}

Anna Schnitzer ist wissenschaftliche Mitarbeiterin am Arbeitsbereich Grundschulpädagogik am Institut für Schulpädagogik und Grundschuldidaktik der Martin-Luther-Universität Halle-Wittenberg. Sie forscht im Bereich Bildungs-, Migrations- und Ungleichheitsforschung u.a. zu Fragen von Mehrsprachigkeit in der Migrationsgesellschaft, Erinnerung im Flucht- und Migrationskontext sowie sozialen Teilhabemöglichkeiten von Kindern und Jugendlichen. Zudem arbeitet sie zu Methoden der qualitativen Sozialforschung, insbesondere biographischen und ethnographischen Ansätzen.

Rebecca Mörgen ist wissenschaftliche Mitarbeiterin (Post-Doc) im Forschungsprojekt „Unbegleitet minderjährige Geflüchtete in institutioneller Betreuung: Chancen und Herausforderungen" am Lehrstuhl für Ausserschulische Bildung und Erziehung am Institut für Erziehungswissenschaft der Universität Zürich. Sie forscht im Bereich der Migrations- und Ungleichheitsforschung u.a. zu Fragen des (institutionellen) Aufwachsens von Kindheit und Adoleszenz, Paradoxien professionellen Handelns im Flucht*Migrationskontext sowie zu sozialen Teilhabemöglichkeiten. Zudem arbeitet sie zu Methoden der qualitativen Sozialforschung, vor allem ethnographisch ausgerichtet und zu multimethodischen Forschungsfragen. 


\section{Kontakt}

Dr. Anna Schnitzer

Institut für Schulpädagogik und Grundschuldidaktik

Frankeplatz 1

D-06110 Halle (Saale)

Tel.: +493455523904

E-Mail: anna.schnitzer@paedagogik.uni-halle.de

URL: https://schulpaed.philfak3.uni-halle.de/grundschule_bereiche_mitarbeiter/gspaed-

fuegd/team/1573810_3243168/

Dr. Rebecca Mörgen

Institut für Erziehungswissenschaft

Universität Zürich

Freiestr. 36

$\mathrm{CH}-8032$ Zürich

Tel.: +41446344563

E-Mail: rmoergen@ife.uzh.ch

URL: https://www.ife.uzh.ch/de/research/abe/mitarbeitende2/moergenrebecca.html

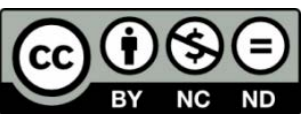

Dieses Werk ist lizenziert unter einer Creative Commons Namensnennung - Weitergabe unter gleichen

Bedingungen 4.0 International Lizenz. 